\title{
Pulmonary arterial hypertension with below threshold pulmonary vascular resistance
}

\author{
Seshika Ratwatte ${ }^{1}$, James Anderson ${ }^{2}$, Geoffrey Strange ${ }^{3,4}$, Carolyn Corrigan $^{5}$, \\ Nicholas Collins ${ }^{6}$, David S. Celermajer ${ }^{7}$, Nathan Dwyer ${ }^{8}$, John Feenstra ${ }^{9}$, \\ Dominic Keating (10 ${ }^{10}$, Eugene Kotlyar ${ }^{5}$, Melanie Lavender ${ }^{11}$, Helen Whitford ${ }^{10}$, \\ Ken Whyte ${ }^{12}$, Trevor Williams ${ }^{10}$, Jeremy P. Wrobel ${ }^{3,11}$, Anne Keogh ${ }^{5}$ and \\ Edmund M. Lau ${ }^{7}$ on behalf of the PHSANZ Registry
}

@ERSpublications

Selected patients with precapillary PH and "borderline" PVR, who fail to meet the current threshold of 3 Wood units, have functional limitation and adverse outcomes, and may potentially benefit from PAH therapy https://bit.ly/2QYaQrC

Cite this article as: Ratwatte S, Anderson J, Strange G, et al. Pulmonary arterial hypertension with below threshold pulmonary vascular resistance. Eur Respir J 2020; 56: 1901654 [https://doi.org/10.1183/ 13993003.01654-2019].

ABSTRACT Pulmonary vascular resistance (PVR) $>3$ Wood units is a criterion of the haemodynamic definition of pulmonary arterial hypertension (PAH). However, this cut-off is conservative and arbitrarily defined. Data is lacking on the natural history, response to therapy and survival of patients diagnosed with precapillary pulmonary hypertension $(\mathrm{PH})$ with mild or borderline elevation of PVR.

In Australia, PAH therapy could be prescribed solely on mean pulmonary arterial pressure (PAP) and pulmonary arterial wedge pressure (PAWP) criteria. Using the Australian and New Zealand Pulmonary Hypertension Registry, we aimed to study a population diagnosed with PAH between January 2004 and December 2017 with the pre-defined haemodynamic characteristics of mean PAP $\geqslant 25 \mathrm{mmHg}$, PAWP $\leqslant 15 \mathrm{mmHg}$ and PVR $<3$ Wood units.

Eighty-two patients met the pre-defined haemodynamic inclusion criteria (mean age $63 \pm 11$ years; 67 females). Underlying aetiologies included idiopathic disease $(n=39)$, connective tissue disease (CTD; $n=42$ ) and HIV infection $(\mathrm{n}=1)$. At diagnosis, mean PAP was $27 \mathrm{mmHg}$ (interquartile range (IQR) 25-30 $\mathrm{mmHg}$ ), PAWP 13 mmHg (IQR 11-14 mmHg) and PVR 2.2 Wood units (IQR 1.9-2.7 Wood units). Baseline 6-min walk distance (6MWD) was $352 \mathrm{~m}$ (IQR 280-416 m) and 77\% of subjects were in New York Heart Association (NYHA) functional class 3 or 4 . All patients were commenced on initial monotherapy with an endothelin receptor antagonist (ERA; $\mathrm{n}=66$ ) or phosphodiesterase type-5 inhibitor (PDE5i; $\mathrm{n}=16$ ). At first re-evaluation, 6MWD increased by $46 \mathrm{~m}$ (IQR 7-96 m) and 35\% of subjects demonstrated improvement in NYHA functional class. After a median follow-up of 65 months (IQR 32-101 months), 18 out of 82 subjects (22.0\%) had died, with estimated 1-year and 5-year survival rates of $98 \%$ and $84 \%$, respectively. Death attributed to PAH occurred in six out of these 18 patients $(33.3 \%, 7 \%$ of total cohort).

Patients with precapillary PH and "borderline" PVR falling outside the current definition have adverse outcomes. Such patients appear to respond to PAH therapy; however, this requires further study in randomised trials.

This article has an editorial commentary: https://doi.org/10.1183/13993003.00962-2020

This article has supplementary material available from erj.ersjournals.com

Received: 21 Aug 2019 | Accepted after revision: 24 March 2020

Copyright OERS 2020 


\section{Introduction}

Pulmonary arterial hypertension (PAH) is a haemodynamic consequence of obstructive remodelling of the small pulmonary arteries and patients can progress to right-heart failure and death [1]. A form of precapillary pulmonary hypertension $(\mathrm{PH}), \mathrm{PAH}$ is traditionally defined by mean pulmonary arterial pressure $(\mathrm{PAP}) \geqslant 25 \mathrm{mmHg}$, pulmonary arterial wedge pressure (PAWP) $\leqslant 15 \mathrm{mmHg}$ and pulmonary vascular resistance $(\mathrm{PVR})>3$ Wood units on right-heart catheterisation (RHC) [2].

Our increasing understanding of the normal ranges of pulmonary haemodynamics has resulted in a recent revision of the definition of PH. At the 6th World Symposium on Pulmonary Hypertension (2018, Nice, France), it was recognised that the original haemodynamic definition of PAH was arbitrary and that the mean PAP threshold should be lowered to $20 \mathrm{mmHg}$, whilst the cut-off values of PAWP $\leqslant 15 \mathrm{mmHg}$ and PVR $>3$ Wood units remained unchanged [3]. Indeed, invasive RHC studies have shown that in healthy subjects normal mean PAP averages $14 \mathrm{mmHg}$ with an upper limit of approximately $20 \mathrm{mmHg}$ [4]. There is also increasing evidence that patients with mildly elevated mean PAP, between $21 \mathrm{mmHg}$ and $24 \mathrm{mmHg}$, experience functional limitation and poorer outcomes compared to those with strictly normal mean PAP $\leqslant 20 \mathrm{mmHg}$ [5-7]. Recent data from large cohorts have confirmed the prognostic significance of mean PAP in the 21-24 mmHg range based on RHC or estimated from transthoracic echocardiography $[8,9]$.

Despite the amended mean PAP value for diagnosis of PAH, the current PVR threshold of 3 Wood units is not based on evidence regarding the normal upper limit for PVR. A meta-analysis of all published data on healthy controls showed that normal PVR remained below 2 Wood units, although the upper limit for elderly patients is less clear due to scarce normative data [4]. Indeed, the limitation of the current PVR threshold was acknowledged at the recent 6th World Symposium [3]. A threshold of 3 Wood units was considered "conservative" but allowed a "safety gap" which would only include patients with clear pulmonary vascular disease $[3,10]$.

Despite the above considerations, it is widely accepted that early treatment of PAH is associated with improved outcomes [11-13].This has led to the recommendation that at-risk groups, such as those with systemic sclerosis or carriers of a BMPR2 mutation, should undergo regular screening for PAH [2]. On the other hand, randomised controlled trials of treatments for $\mathrm{PAH}$ have universally included patients with moderate to severe elevation of PVR $[2,14]$. Hence, there is little or no published data on patients with precapillary PH with only "borderline" or mild elevation of PVR. The natural history and long-term prognosis of these patients are unclear but important, as they may represent a substantial group of symptomatic patients. Furthermore, it is unknown whether these patients respond to targeted PAH therapy and this question will be difficult to answer in the current era where treatments are only recommended if PVR exceeds 3 Wood units.

Using data from the Pulmonary Hypertension Society of Australia and New Zealand (PHSANZ) Registry, the aim of the present study was to describe the clinical characteristics, treatment response to PAH therapy and long-term outcomes of patients with "early" precapillary PH. This was facilitated by examining a group of patients in Australia and New Zealand where PVR was not part of the haemodynamic definition required for treatment initiation in our region. We thus prospectively defined this group of interest with the haemodynamic characteristics of mean PAP $\geqslant 25 \mathrm{mmHg}$, PAWP $\leqslant 15 \mathrm{mmHg}$ and PVR $<3$ Wood units.

\section{Methods}

We performed a retrospective observational study using the PHSANZ Registry (which commenced in December 2011 and currently has 21 participating centres across Australia and New Zealand). Although the PHSANZ Registry enrolls patients with all groups of $\mathrm{PH}$, the largest population is Group $1 \mathrm{PAH}$ [15]. Data for patients diagnosed prior to December 2011 were entered retrospectively, whilst data for incident cases and follow-up data were entered prospectively using a dedicated software platform (FileMaker Pro 17, Claris, Santa Clara, USA). The PHSANZ Registry records demographics, detailed data of investigations (invasive haemodynamic tests, pulmonary function tests (PFTs) and echocardiographic data), functional

Affiliations: ${ }^{1}$ Dept of Cardiology, Concord Repatriation and General Hospital, Concord, Australia. ${ }^{2}$ Respiratory Dept, Sunshine Coast University Hospital, Birtyna, Australia. ${ }^{3}$ School of Medicine, University of Notre Dame, Fremantle, Australia. ${ }^{4}$ Pulmonary Hypertension Society of Australia and New Zealand. ${ }^{5}$ Heart Transplant Unit, St Vincent's Hospital, Darlinghurst, Australia. ${ }^{6}$ Dept of Cardiology, John Hunter Hospital, Newcastle, Australia. ${ }^{7}$ Sydney Medical School, University of Sydney, Camperdown, Australia. ${ }^{8}$ Dept of Cardiology, Royal Hobart Hospital, Hobart, Australia. ${ }^{9}$ Queensland Lung Transplant Service, Prince Charles Hospital, Chermside, Australia. ${ }^{10}$ Dept of Allergy, Immunology and Respiratory Medicine, The Alfred Hospital, Melbourne, Australia.

${ }^{11}$ Advanced Lung Disease Unit, Fiona Stanley Hospital, Murdoch, Australia. ${ }^{12}$ Greenlane Clinical Centre Auckland City Hospital, Auckland, New Zealand.

Correspondence: Edmund Lau, Dept of Respiratory Medicine, Royal Prince Alfred Hospital, Missendon Road, Camperdown 2050, Australia. E-mail: edmund.laudasydney.edu.au 
capacity (New York Heart Association (NYHA) functional class and six-min walk distance (6MWD)), treatment data and outcome data. Data was collected at time of enrolment and at subsequent periodic reviews determined by the treating physician (typically every 3-6 months).

Using the PHSANZ Registry, patients with physician diagnosed Group 1 PAH fulfilling the following haemodynamic characteristics (mean PAP $\geqslant 25 \mathrm{mmHg}$, PAWP $\leqslant 15 \mathrm{mmHg}$ and PVR $<3$ Wood units) were included in the present study. It is important to note that the old haemodynamic definition of PAH (mean PAP $\geqslant 25 \mathrm{mmHg}$ and PAWP $\leqslant 15 \mathrm{mmHg}$ without the PVR $>3$ Wood units criterion) was used for the PHSANZ Registry, as data collection began prior to the official inclusion of PVR $>3$ Wood units into the haemodynamic definition of PAH at the 5th World Symposium on Pulmonary Hypertension in 2013. The diagnosis of Group $1 \mathrm{PAH}$ was made by the treating physician at each centre after the integration of all clinical, radiological and haemodynamic data. Patients with $\mathrm{PH}$ deemed to be caused by left to right cardiac shunt, congenital heart disease, chronic liver disease, lung disease and left-heart disease, as well as chronic thromboembolic disease, were excluded from the analysis. Baseline demographics, disease aetiology, comorbidities, NYHA functional class, $6 \mathrm{MWD}$, lung function tests and invasive haemodynamics were recorded, as well as the type of PAH therapy administered. Mortality and last follow-up were censored at March 01, 2017. The PHSANZ Registry protocol was approved by the human ethical committee at St. Vincent's Hospital, Sydney, Australia (HREC: LNR/11/SVH/178).

Variables were expressed as mean \pm standard deviation (SD) or median (interquartile range (IQR)) if their distribution was non-parametric. All-cause mortality data was determined from the database and patients were censored at the date of last follow-up. Mortality status was updated to the PHSANZ Registry at each clinical follow-up. The Kaplan-Meier method was use to estimate survival, with censoring at the date of last follow-up. Risk assessment was performed using the REVEAL 2.0 Risk Score and the modified three tier low, intermediate and high risk strata [16]. This REVEAL 2.0 Risk Score has recently been validated in the PHSANZ PAH population [16, 17]. The Cox model was used to determine baseline variables predictive of survival. For all analyses, a two-tailed p-value of less than 0.05 was deemed to be significant. Statistical analyses were conducted using STATA version 15.1 (StataCorp LLC, College Station, TX, USA).

\section{Results}

\section{Study population}

At the time of data extraction, there were 3292 patients in the PHSANZ registry. Of these, 2378 were diagnosed as having Group $1 \mathrm{PAH}$. Of these, 90 patients met the haemodynamic criteria for inclusion (mean PAP $\geqslant 25 \mathrm{mmHg}$, PAWP $\leqslant 15 \mathrm{mmHg}$ and PVR $<3$ Wood units). However, eight of these were excluded as their $\mathrm{PH}$ was due to congenital heart disease with left to right shunt $(\mathrm{n}=4)$, left heart disease or pulmonary disease $(\mathrm{n}=4)$ (figure 1 ).

Thus, 82 patients were included in the present study, with a mean age of $63 \pm 11$ years, of which 67 (82\%) were female. Baseline characteristics are summarised in table 1. Underlying diagnoses included 40 patients

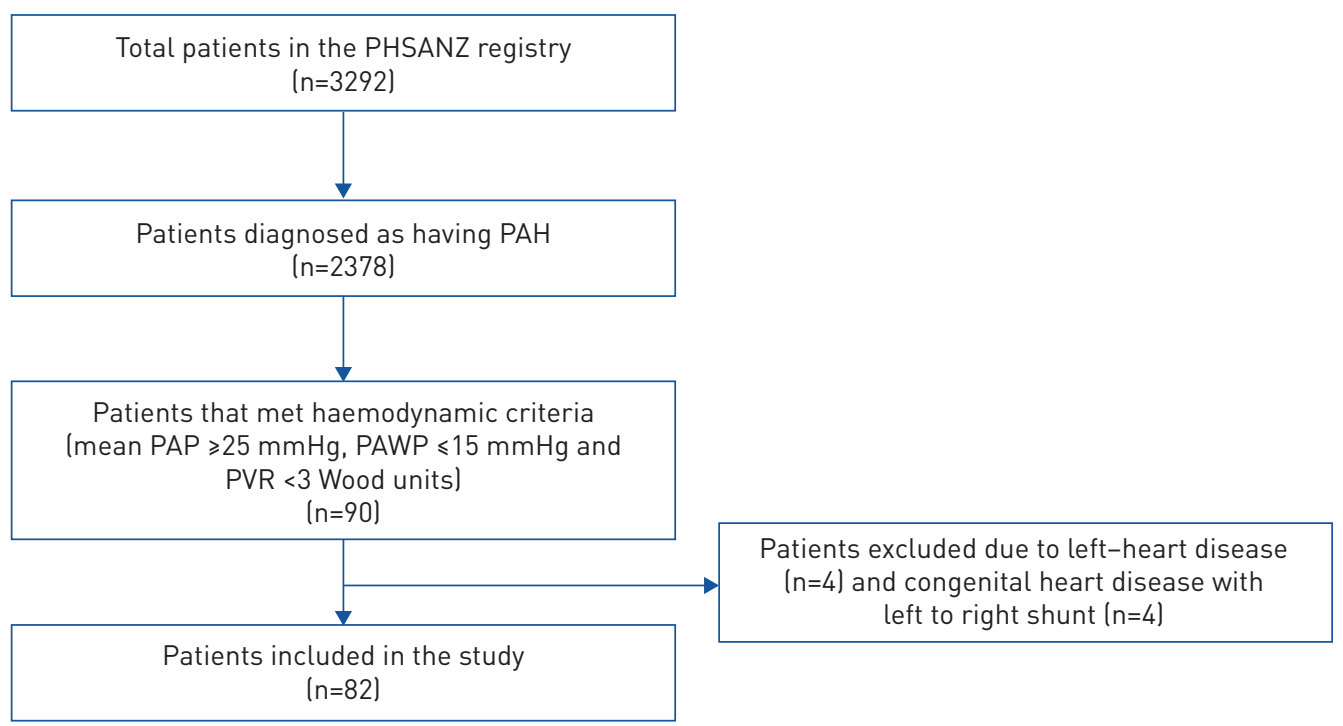

FIGURE 1 Flow diagram of the study population from the Pulmonary Hypertension Society of Australia and New Zealand (PHSANZ) registry. PAH: pulmonary arterial hypertension; PAP: pulmonary arterial pressure; PAWP: pulmonary arterial wedge pressure; PVR: pulmonary vascular resistance. 
TABLE 1 Baseline characteristics of the study population

\begin{tabular}{|c|c|}
\hline Characteristic & Result \\
\hline Age years & $63 \pm 11$ \\
\hline Female gender $\%$ & 82 \\
\hline BMI kg. $\mathrm{m}^{-2}$ & $31.4 \pm 7.8$ \\
\hline \multicolumn{2}{|l|}{ Aetiology } \\
\hline Non-CTD associated PAH & 40 \\
\hline Idiopathic & 39 \\
\hline HIV associated & 1 \\
\hline CTD associated PAH & 42 \\
\hline Systemic sclerosis spectrum & 39 \\
\hline Rheumatoid arthritis & 2 \\
\hline Systemic lupus erythematosus & 1 \\
\hline \multicolumn{2}{|l|}{ Comorbidities } \\
\hline Diabetes mellitus & $20(24)$ \\
\hline Hypertension & $45(55)$ \\
\hline Dyslipidaemia & $36(44)$ \\
\hline Ischaemic heart disease & $22(27)$ \\
\hline Peripheral vascular disease & $5(6)$ \\
\hline Current smoker & $3(4)$ \\
\hline Ex-smoker & $26(32)$ \\
\hline OSA & $20(24)$ \\
\hline \multicolumn{2}{|l|}{ Baseline haemodynamics } \\
\hline Mean PAP mmHg & $27(25-30)$ \\
\hline PAWP $\mathrm{mmHg}$ & $13(11-14)$ \\
\hline PVR Wood units & $2.2(1.9-2.7)$ \\
\hline Cardiac output L.min ${ }^{-1}$ & $6.7(5.8-7.9)$ \\
\hline Cardiac index $\mathrm{L} \cdot \mathrm{min}^{-1} \cdot \mathrm{m}^{-2}$ & $3.55(3.1-4.2)$ \\
\hline RAP $\mathrm{mmHg}$ & $8.0(5.2-10.0)$ \\
\hline Mean SAP mmHg & $98(83-106)$ \\
\hline SVR dynes.sec $\cdot \mathrm{cm}^{-5}$ & $1078(852-1240)$ \\
\hline $\mathrm{HR}$ beats $\cdot \mathrm{min}^{-1}$ & 72 (67-87) \\
\hline Baseline 6MWD m & $352(280-416)$ \\
\hline \multicolumn{2}{|l|}{ PFT } \\
\hline $\mathrm{FEV}_{1} \%$ predicted & $91(75-96)$ \\
\hline FVC \% predicted & 90 (81-105) \\
\hline TLC \% predicted & $93(80-98)$ \\
\hline$D_{\text {Lco }} \%$ predicted & $53(45-62)$ \\
\hline \multicolumn{2}{|l|}{ NYHA functional class } \\
\hline 1 & $1(1)$ \\
\hline 2 & $19(23)$ \\
\hline 3 & $60(73)$ \\
\hline 4 & 2 (3) \\
\hline
\end{tabular}

Data are presented as $n, n(\%)$, mean \pm SD or median (IQR), unless otherwise stated. BMI: body mass index; CTD: connective tissue disease; PAH: pulmonary arterial hypertension; OSA: obstructive sleep apnoea; PAP: pulmonary arterial pressure; PAWP: pulmonary arterial wedge pressure; PVR: pulmonary vascular resistance; RAP: right-atrial pressure; SAP: systemic arterial pressure; SVR: systemic vascular resistance; HR: heart rate; 6MWD: 6-min walk distance; PFT: pulmonary function test; $\mathrm{FEV}_{1}$ : forced expiratory volume in $1 \mathrm{~s}$; FVC: forced vital capacity; TLC: total lung capacity; $D_{\mathrm{LCO}}$ : diffusing capacity of the lung for carbon monoxide; NYHA: New York Heart Association.

with non-connective tissue disease (CTD)-associated PAH (idiopathic $(n=39)$ and human immunodeficiency disease $(\mathrm{n}=1))$ and 42 patients with CTD-associated PAH (systemic sclerosis spectrum disease $(n=39)$, rheumatoid arthritis $(n=2)$ and systemic lupus erythematosus $(n=1))$. At diagnosis, baseline haemodynamics showed mild PH with mean PAP $27 \mathrm{mmHg}$ (IQR 25-30 mmHg), PAWP $13 \mathrm{mmHg}$ (IQR 11-14 mmHg) and PVR 2.2 Wood units (IQR 1.9-2.7 Wood units). The median 6MWD at baseline was $352 \mathrm{~m}$ (IQR $280-416 \mathrm{~m}$ ). The majority of patients had significant functional limitation at diagnosis as demonstrated by NYHA functional class status. Using the Reveal 2.0 risk score, $61 \%$ of the cohort was low-risk, 35\% were intermediate risk and $4 \%$ were high-risk at diagnosis. Baseline characteristics of the non-CTD and CTD groups were broadly similar apart from a higher prevalence of diabetes, lower 6MWD and more severe NYHA functional class in the non-CTD group (supplementary table S1). 
Pulmonary arterial hypertension therapy

All patients were commenced on initial monotherapy. The mean time between confirmation of diagnosis by RHC and commencement of PAH treatment was $30 \pm 38$ days. Sixty-six patients were commenced on an endothelin receptor antagonist (ERA) and 16 were commenced on a phosphodiesterase type-5 inhibitor (PDE5i) at the discretion of the treating physician. Of the patients commenced on an ERA, 50 were commenced on bosentan, seven on sitaxsentan, five on macitentan and four on ambrisentan. The majority of patients started on PDE5i's were started on sildenafil $(n=15)$, with one patient commenced on tadalafil. All patients commenced on sitaxsentan were switched to alternate endothelin antagonist medications when this drug was taken off the market in Australia. There were 14 patients (17\%) who were escalated to combination therapy with both a PDE5i and an ERA during the follow up period. There were no treatment interruptions due to side effects and all patients remained on treatment until the end of the follow-up period. Types of PAH treatment administered are summarised in table 2 . Treatment stratified by underlying aetiology can be found in supplementary table S2.

\section{Treatment response}

Treatment response was assessed at least 3 months after treatment commencement. Median time of first post-treatment evaluation was 5 months (IQR 4-12 months). In the overall population, 6MWD increased by $46 \mathrm{~m}$ (IQR $7-96 \mathrm{~m})(\mathrm{p}=0.01)$ and $35 \%$ of patients demonstrated improvement in NYHA functional class status (figures $2 \mathrm{a}$ and $2 \mathrm{~b}$ ). Using the REVEAL 2.0 risk score, PAH therapy increased the proportion of patients in the low-risk category from $61 \%$ to $72 \%$ (figure 3 ).

Treatment response stratified by underlying aetiology showed that patients with non-CTD-associated PAH demonstrated a change in 6MWD of $65 \mathrm{~m}$ (IQR 14-104 m) $(\mathrm{p}=0.03)$, whereas the CTD group changed by $41 \mathrm{~m}$ (IQR 8-85 m) ( $\mathrm{p}=0.15$ ) (supplementary figure S1). Similarly, 34\% of patients with non-CTD-associated PAH showed improvement in NYHA functional class versus $20 \%$ for CTD-associated PAH (supplementary figures S2a and S2b). Using the REVEAL 2.0 risk score, PAH therapy resulted in a similar proportion of improvement into the lower-risk category at time of first follow-up when stratified by aetiology (supplementary figures S3a and S3b).

Long term follow-up

Median follow-up after diagnosis was 65 months (IQR 32-101 months) for the entire cohort. Follow-up RHC was not mandated by the PHSANZ Registry but was performed at the discretion of the treating

\section{TABLE 2 Therapy at diagnosis and last follow-up}

\section{Strategy}

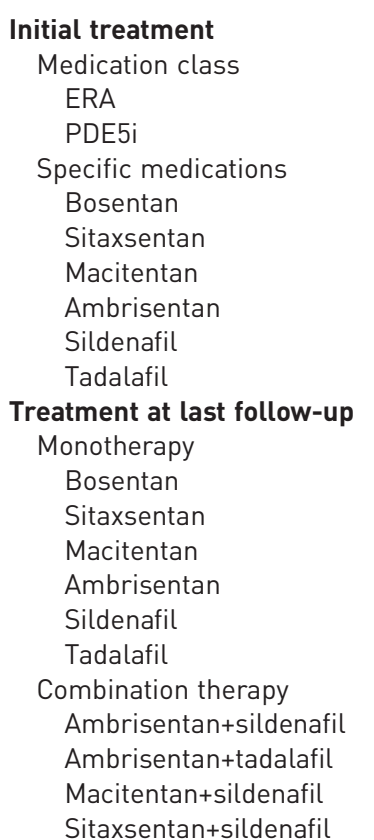

$66(80)$
$16(20)$
$50(61)$
$7(9)$
$5(6)$
$4(5)$
$15(18)$
$1(1)$

$68(83)$
$38(46)$
$1(1)$
$8(10)$
$4(5)$
$14(17)$
$3(4)$
$14(17)$
$8(10)$
$1(1)$
$4(5)$
$1(1)$

ERA: endothelin receptor antagonist; PDE5i: phosphodiesterase type-5 inhibitor. 

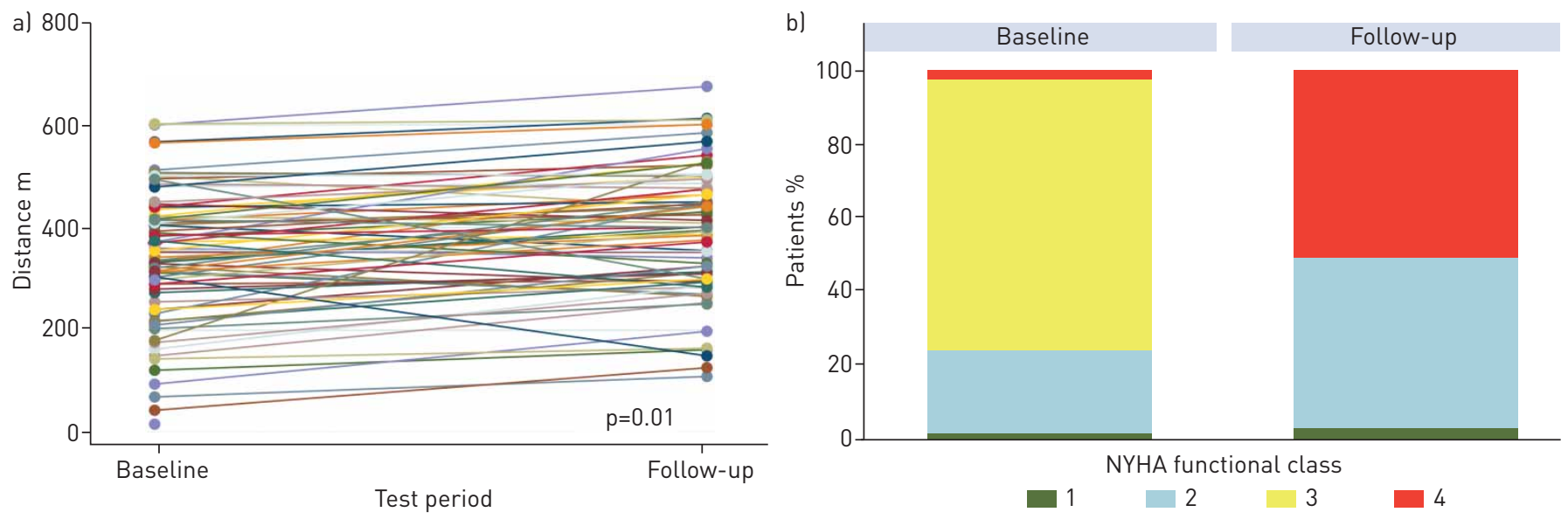

FIGURE 2 (a) Change in 6-min walk distance (6MWD) between baseline and first follow-up after initial treatment initiation (median improvement was $46 \mathrm{~m}$ (IQR 7-96 m); p=0.01). (b) Change in New York Heart Association (NYHA) functional class between baseline and first follow-up (35\% of patients had an improvement in functional class status). IQR: interquartile range.

physician. In order to evaluate long-term progression of PAH, we only included follow-up RHCs which were performed at least 1 year after initial diagnosis. Twenty-six out of 82 subjects (CTD group $(n=18)$ and non-CTD group $(\mathrm{n}=8)$ ) had long-term follow-up RHC available at a median follow-up time of 48 months (IQR 32-58 months). Overall, there were no significant changes in mean PAP and PVR between RHC at diagnosis and at long-term follow-up (supplementary table S3). Of note, seven out of 26 patients (26.9\%) developed PVR $>3$ Wood units at follow-up RHC. In these patients, PVR increased from 2.6 Wood units (IQR 2.3-2.8 Wood units) to 3.4 Wood units (IQR 3.2-4.6 Wood units).

There were 29 individual hospitalisations related to management of PAH in our cohort, which correlated to a hospitalisation rate of $6.3 \%$ per year. Six patients had recurrent hospitalisations during the follow-up period.

There were 18 deaths (22\%) during the study period and PAH was identified as the likely cause of death (sudden cardiac death or right-ventricular failure) in six patients (33\%). The three patients who died from right-ventricular failure had repeated hospitalisations for PAH prior to death. Of the remaining 12 deaths, three were from unknown causes and nine were attributed to causes unrelated to PAH (table 3). KaplanMeier estimates of 1-year, 3-year and 5-year survival were 0.99 (95\% confidence interval (CI) $0.92-1.00$ ), 0.89 (95\% CI $0.79-0.94)$ and 0.84 (95\% CI 0.73-0.91), respectively (figure 4a). There was no significant difference in survival between the non-CTD group versus the CTD group $(p=0.85)$ (figure $4 \mathrm{~b}$ ). On use of a univariate Cox model, the presence of comorbidities (ischaemic heart disease, diabetes, hypertension and obesity) were not predictive of survival ( $\mathrm{p}>0.15$ for all).

\section{Discussion}

To the best of our knowledge, this is the first study to examine the characteristics, treatment outcomes and survival of a PAH population with borderline elevation of PVR. This was possible because of a historical

FIGURE 3 REVEAL 2.0 modified risk assessment at baseline and first follow-up after initial treatment initiation (the proportion of patients in the low risk category increased from $61 \%$ to $72 \%$ ).

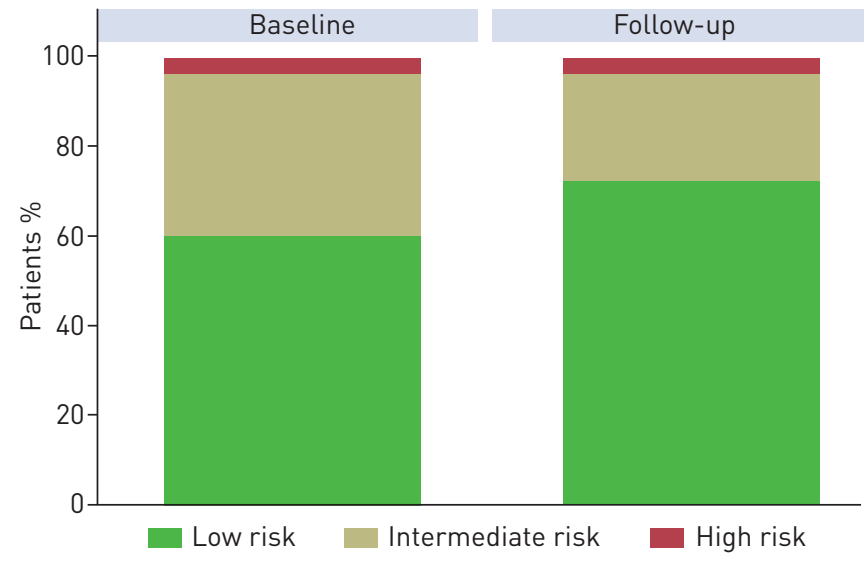




\begin{tabular}{lr} 
TABLE 3 Cause of death during follow-up & $\mathbf{n}(\%)$ \\
\hline Cause & $18(100)$ \\
\hline Total deaths & $6(33)$ \\
Deaths attributed to PAH & $3(17)$ \\
Right-ventricular failure & $3(17)$ \\
Sudden cardiac event & $9(50)$ \\
Deaths not directly attributed to PAH & $2(11)$ \\
Respiratory failure & $3(17)$ \\
Acute respiratory infection & $3(17)$ \\
Malignancy & $1(6)$ \\
Other & $3(17)$ \\
Unknown &
\end{tabular}

PAH: pulmonary arterial hypertension.

"gap" (prior to 2013) in which there was no accepted PVR criterion required for the definition of PAH and hence the PHSANZ Registry enrolled patients with sub-criterion PVR values. These patients, with precapillary PH but PVR $<3$ Wood Units, displayed significant functional limitation at baseline and appeared to respond to PAH therapy with both an improvement in walk distance, NYHA functional class and global risk assessment. Despite this apparently favourable treatment response mortality during follow-up was significant, with an estimated 5-year survival rate of $84 \%$; whereas age and gender matched subjects from the general adult population in Australia would have an expected life-expectancy of 85 years [18]. Furthermore, progressive PAH was the cause of death in $33 \%$ of patients who died during the follow-up period, suggesting that this is not a benign condition despite only mild elevation of PVR at diagnosis. The current haemodynamic definition of PAH, which requires PVR $>3$ Wood units, may potentially "miss" patients with clinically important pulmonary vascular disease who are at increased risk of adverse outcomes and who may benefit from early PAH therapy.

Expert consensus from the recent 6th World Symposium on Pulmonary Hypertension recommended that a PVR cut-off $>3$ Wood units should remain for the haemodynamic diagnosis of PAH. However, prior studies have demonstrated that PVR remains $<2$ Wood units in healthy subjects $[4,19]$. Thus, the current PVR $>3$ Wood units cut-off is intended to allow greater specificity for the diagnosis of PAH but at the expense of sensitivity, particularly in the younger population. It is noteworthy that the traditional definition of $\mathrm{PH}$ requiring mean $\mathrm{PAP} \geqslant 25 \mathrm{mmHg}$ has recently been modified to $20 \mathrm{mmHg}$, which is aligned with what is known regarding the upper limit of normal mean PAP. Furthermore, the impetus for lowering the mean PAP threshold is supported by recent studies showing that patients with systemic

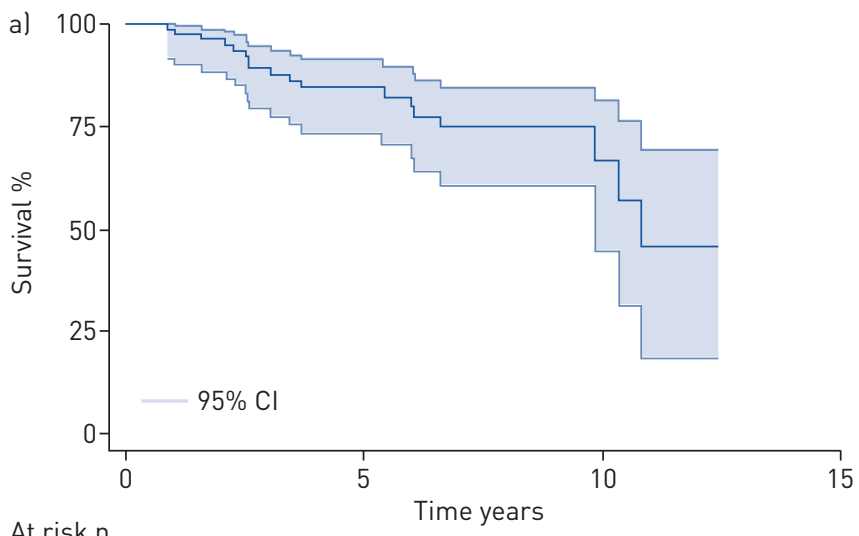

82
40
8

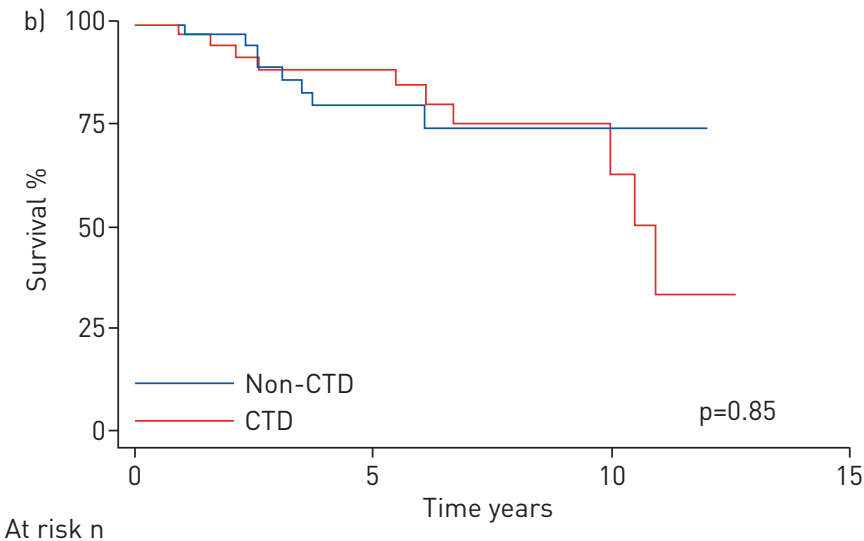

0 Non-CTD 40

CTD 42
18

22
0

FIGURE 4 (a) Kaplan-Meier survival estimates from time of pulmonary arterial hypertension (PAH) diagnosis for the entire study cohort (1-year, 3-year and 5-year survival estimates were 0.99 (95\% Cl 0.92-1.00), 0.89 (95\% Cl 0.79-0.94) and 0.84 (95\% Cl 0.73-0.91), respectively). (b) KaplanMeier survival estimates from time of PAH diagnosis stratified by aetiology (connective tissue disease (CTD) related PAH and non-CTD related $\mathrm{PAH}$. $\mathrm{Cl}$ : confidence interval. 
sclerosis and mildly elevated mean PAP (21-24 mmHg) have more functional limitation and increased risk of disease progression compared to those with mean PAP $<20 \mathrm{mmHg}[5,20,21]$. In addition, large population-based RHC studies have found that patients with mean PAP in the $21-24 \mathrm{mmHg}$ range have excess mortality and hospitalisations compared to those with lower ranges of mean PAP [9]. Therefore, it can be argued that the current PVR threshold of 3 Wood units is not based on available evidence and does not represent the normal upper limit of PVR in healthy subjects [4]. Further studies are required to determine the threshold of PVR elevation that is associated with increased risk of adverse outcomes.

Despite relatively mild haemodynamic impairment in the group studied, most patients presented with an advanced NYHA functional class, although comorbidity status may impact on this tendency towards more advanced functional class. For example, our population displayed a high proportion of cardiometabolic risk factors, such as ischaemic heart disease, hypertension, diabetes and obesity. Thus, it is possible that poor functional capacity may have other contributory factors beyond pulmonary haemodynamics and right-heart function. Invasive exercise haemodynamics were not performed and it is unknown whether these patients developed a brisk rise in PAP during exercise, which could account for the advanced functional disability experienced despite mild resting haemodynamic derangement [22].

The patients in our cohort had high normal PAWP values (median $13 \mathrm{mmHg}$ ) but did not have overt left-heart disease (as deemed by their treating physician). While we cannot fully exclude the possibility that some of these patients had occult left-heart disease, the PAWP in all subjects remained below $15 \mathrm{mmHg}$ which is in line with the current haemodynamic classification of precapillary PH. Assessment of left-heart filling pressure following fluid loading or exercise challenge would have allowed greater confidence in excluding left-heart disease in our subjects $[23,24]$. Reassuringly, none of these patients had $\mathrm{PAH}$ treatment withdrawn due to worsening symptoms, which can commonly occur in the setting of left-heart disease [25]. Our cohort highlights the real-world diagnostic challenge of differentiating occult left-heart disease (especially heart failure with preserved ejection fraction) from $\mathrm{PAH}$.

The estimated survival rate for this cohort was $84 \%$ at 5 years and is thus higher than the 5 -year survival rate of 57\% reported in the REVEAL registry [26] and the more contemporary 3-year survival rate of $77 \%$ reported from the PHSANZ registry in 2018 [27]. It should be noted, however, that the latter figure included only those with idiopathic, heritable and drug-induced PAH. The better survival seen in our study population is not unexpected in a population with a lesser degree of haemodynamic abnormality at diagnosis. However, six out of 18 patients (33\%) who died during the follow-up period died from a cause that was attributable to PAH. This is despite the fact that all patients received PAH therapy following diagnosis and showed improvements in 6MWD, NYHA functional class and risk assessment at first follow-up. Whilst these patients did not have repeat RHC close to their death, all patients who died of progressive right-heart failure had recurrent hospitalisations related to PAH. This supports the notion that PAH can be a progressive disease, even when diagnosed at a stage with apparently only mild haemodynamic impairment, and highlights the importance of early diagnosis and screening of "at risk" populations, such as those with systemic sclerosis [28].

Only a small proportion of patients (26 out of 82 ) had follow-up RHC at long term follow-up, since RHC was not mandated by the Registry and was performed at the discretion of the treating physician. Of those who did have a long-term follow-up RHC, there was no significant change in mean PAP and PVR. However, all patients had received PAH therapy so this data does not strictly inform the natural history of untreated patients with borderline PVR elevation. It is of note that seven out of 26 patients with follow-up RHC crossed the threshold of PVR $>3$ Wood units at follow-up, supporting the notion that at least a proportion of patients are at risk of progression despite $\mathrm{PAH}$ therapy.

Our study characterises a group of patients with borderline PVR elevation who may not fulfil the current haemodynamic definition of PAH but may still experience adverse outcomes. However, lowering of the current PVR threshold can potentially lead to the problem of over-diagnosis, particularly when non-efficacious or potentially harmful therapy is given to patients without true pulmonary vascular disease. As a sensitivity analysis, we compared the survival of the current study cohort (PVR $<3$ Wood units) with PAH patients from the PHSANZ registry who had PVR 3-4 Wood units, mean PAP $\geqslant 25 \mathrm{mmHg}$ and PAWP $\leqslant 15 \mathrm{mmHg}$. There was no significant difference in survival between the two groups (supplementary figure S4) and this provides some reassurance that an excess harmful signal was not observed when PAH therapy was administered in our study population. It is important to emphasise that all our patients were diagnosed with $\mathrm{PAH}$ and were commenced on PAH therapy by clinicians experienced in the management of $\mathrm{PH}$. The findings of the present study cannot be extrapolated to all patients with this haemodynamic profile, where $\mathrm{PH}$ could be due to other causes such as lung disease, chronic thromboembolic disease or occult left-heart disease.

There are several limitations to this study. It was observational in nature and based on a relatively small number of patients, the majority of which had underlying CTD which is a strong risk factor for PAH. The 
patients included in the current study were given a diagnosis of PAH by their treating physicians and no central review of diagnosis was required in the PHSANZ Registry. Only patients who were commenced on therapy were included in the PHSANZ registry. Thus, we cannot provide a comparator group with similar haemodynamic profiles where PAH therapy was not administered. Provocative testing with fluid or exercise challenge was not performed during diagnostic RHC and this could have provided additional insights into the haemodynamic phenotyping of this population. Furthermore, diuretic therapy was not captured in the registry, so we were unable to comment on whether diuretic therapy accounted, at least in part, for the improvement demonstrated at first follow-up. Due to the fact that follow-up RHC was not mandated by the PHSANZ Registry, long term follow-up RHC was only available in a minority of patients. Systematic follow-up RHC would have allowed comprehensive evaluation of patients with disease progression and the determination of possible risk factors that predict such progression. Finally, the precise mechanism of death was not known in a proportion of our patients, as our registry was not linked to the national death registry. Although our cohort demonstrated clinical response to PAH therapy, this was only observational in nature. There are currently no randomised, controlled data at present to support treating patients with borderline elevation of PVR.

\section{Conclusions}

Certain patients with precapillary PH who fail to meet the PVR threshold of 3 Wood units may have adverse clinical outcomes and may potentially benefit from PAH therapy. Survival data shows a significant proportion of patients died of a PAH-related cause during follow-up, highlighting that this is not a benign condition. Further studies are needed to define the PVR thresholds which impact on long-term prognosis and whether early treatment of patients with borderline elevation of PVR confers beneficial impact on symptoms, exercise capacity and/or adverse clinical events.

Conflict of interest: J. Anderson reports research support from GlaxoSmithKline and travel grants from Actelion and Bayer. G. Strange reports grants from Actelion, Bayer, Pfizer Pharmaceuticals and GlaxoSmithKline, and personal fees from Actelion, Arena Pharmaceuticals, Bayer and GlaxoSmithKline, outside the submitted work. C. Corrigan has nothing to disclose. N. Collins reports fees for lectures from Actelion, outside the submitted work. D.S. Celermajer has nothing to disclose. N. Dwyer has participated in advisory board work for Actelion, GlaxoSmithKline and Bayer, outside the submitted work. J. Feenstra has nothing to disclose. D. Keating reports personal fees for lectures from GlaxoSmithKline, Actelion and Novartis, outside the submitted work. E. Kotlyar reports personal fees for lectures from Actelion, Bayer, Novartis, Servier and GlaxoSmithKline, as well as advisory board work for GlaxoSmithKline, Actelion and Bayer, outside the submitted work. M. Lavender has been consultant to and received grants from GlaxoSmithKline, Actelion and Bayer, outside the submitted work. H. Whitford reports fees for lectures, advisory board work and travel grants from Actelion, GlaxoSmithKline and Bayer, outside the submitted work. K. Whyte has nothing to disclose. T. Williams reports advisory board work for Actelion, GlaxoSmithKline and Bayer, as well as grants from Actelion, outside the submitted work. J.P. Wrobel reports fees for lectures, advisory board work and travel grants from Actelion, Boehringer Ingelheim, GlaxoSmithKline and Roche, outside the submitted work. A. Keogh reports advisory board work for Actelion, GlaxoSmithKline and Bayer, as well as grants from Actelion, Bayer, Pfizer, Gilead, Arena, Respira, Acceleron, Pfizer and Bellerophon, outside the submitted work. E.M. Lau reports speaking and consultancy fees from Actelion and Menarini Pharmaceuticals, as well as research support from Actelion and GlaxoSmithKline. S. Ratwatte has nothing to disclose.

Support statement: Funding support for the Pulmonary Hypertension Society of Australia and New Zealand (PHSANZ) Registry was provided by Actelion Pharmaceuticals, Allied Healthcare, Bayer, GlaxoSmithKline, Novartis and Pfizer. GlaxoSmithKline provided research funding support for the primary author, but were not involved in conceptualisation, analysis or manuscript preparation. Funding information for this article has been deposited with the Crossref Funder Registry.

\section{References}

1 Humbert M, Guignabert C, Bonnet S, et al. Pathology and pathobiology of pulmonary hypertension: state of the art and research perspectives. Eur Respir J 2019; 53: 1801887.

2 Galiè N, Humbert M, Vachiery JL, et al. 2015 ESC/ERS Guidelines for the diagnosis and treatment of pulmonary hypertension. Eur Respir J 2015; 46: 903-975.

3 Simonneau G, Montani D, Celermajer DS, et al. Haemodynamic definitions and updated clinical classification of pulmonary hypertension. Eur Respir J 2019; 53: 1801913.

4 Kovacs G, Olschewski A, Berghold A, et al. Pulmonary vascular resistance during exercise in normal subjects: a systematic review. Eur Respir J 2012; 39: 319-328.

5 Valerio CJ, Schreiber BE, Handler CE, et al. Borderline mean pulmonary artery pressure in patients with systemic sclerosis: transpulmonary gradient predicts risk of developing pulmonary hypertension. Arthritis Rheum 2013; 65: 1074-1084.

6 Coghlan JG, Wolf M, Distler O, et al. Incidence of pulmonary hypertension and determining factors in patients with systemic sclerosis. Eur Respir J 2018; 51: 1701197.

7 Douschan P, Kovacs G, Avian A, et al. Mild elevation of pulmonary arterial pressure as a predictor of mortality. Am J Respir Crit Care Med 2018; 197: 509-516.

8 Strange G, Stewart S, Celermajer DS, et al. Threshold of pulmonary hypertension associated with increased mortality. J Am Coll Cardiol 2019; 73: 2660-2672. 
9 Maron B, Hess E, Maddox T, et al. Association of borderline pulmonary hypertension with mortality and hospitalization in a large patient cohort: insights from the Veterans Affairs Clinical Assessment, Reporting, and Tracking Program. Circulation 2016; 133: 1240-1248.

10 Galiè N, McLaughlin VV, Rubin L, et al. An overview of the 6th World Symposium of Pulmonary Hypertension. Eur Respir J 2019; 53: 1802148.

11 Lau EM, Manes A, Celermajer DS, et al. Early detection of pulmonary vascular disease in pulmonary arterial hypertension: time to move forward. Eur Heart J 2011; 20: 2489-2498.

12 Lau EM, Humbert M, Celermajer DS. Early detection of pulmonary arterial hypertension. Nat Rev Cardiol 2015; 12: 143-155.

13 Humbert M, Yaici A, de Groote P, et al. Screening for pulmonary arterial hypertension in patients with systemic sclerosis: clinical characteristics at diagnosis and long-term survival. Arthritis Rheum 2011; 63: 3522-3530.

14 Galiè N, Barberà JA, Frost A, et al. Initial use of ambrisentan plus tadalafil in pulmonary arterial hypertension. N Engl J Med 2015; 373: 834-844.

15 Strange G, Playford D, Stewart S, et al. Pulmonary hypertension: prevalence and mortality in the Armadale Echocardiography Cohort. Heart 2012; 98: 1805-1811.

16 Benza RL, Gomberg-Maitland M, Elliott CG, et al. Predicting survival in patients with pulmonary arterial hypertension: REVEAL risk score calculator 2.0 and comparison with ESC/ERS-based risk assessment strategies. Chest 2019; 156: 323-337.

17 Anderson JJ, Lau EM, Lavender M, et al. Restrospective validation of the REVEAL 2.0 risk score with the Australian and New Zealand Pulmonary Hypertension Registry Cohort. Chest 2020; 157: 162-172.

18 Australian Institute of Health and Welfare. www.aihw.gov.au/reports/life-expectancy-death/deaths-in-australia/ contents/lifeexpectancy Date last accessed: June 10, 2020. Date last updated: July 17, 2019.

19 Ekelund LG, Holmgren A. Central hemodynamics during exercise. Circulat Res 1967; 20: 133-143.

20 Bae S, Saggar R, Bolster MB, et al. Baseline characteristics and follow-up in patients with normal haemodynamics versus borderline mean pulmonary arterial pressure in systemic sclerosis: results from the PHAROS registry. Ann Rheum Dis 2012; 71: 1335-1342.

21 Kovacs G, Maier R, Aberer E, et al. Borderline pulmonary arterial pressure is associated with decreased exercise capacity in scleroderma. Am J Respir Crit Care Med 2009; 180: 881-886.

22 Kovacs G, Herve P, Barbera JA, et al. An official European Respiratory Society statement: pulmonary haemodynamics during exercise. Eur Respir J 2017; 50: 1700578.

23 Vachiéry J, Tedford RJ, Rosenkranz S, et al. Pulmonary hypertension due to left heart disease. Eur Respir J 2019; 53: 1801897.

24 Borlaug B, Nishimura RA, Sorajja P, et al. Exercise hemodynamics enhance diagnosis of early heart failure with preserved ejection fraction. Circ Heart Fail 2010; 3: 588-595.

25 Vachiéry J, Delcroix M, Al-Hiti H, et al. Macitentan in pulmonary hypertension due to left ventricular dysfunction. Eur Respir J 2018; 51: 1701886.

26 McGoon MD, Miller DP. REVEAL: a contemporary US pulmonary arterial hypertension registry. Eur Respir Rev 2012; $21: 8-18$

27 Strange G, Lau EM, Giannoulatou E, et al. Survival of idiopathic pulmonary arterial hypertension patients in the modern era in Australia and New Zealand. Heart Lung Circ 2018; 27: 1368-1375.

28 Xanthoui P, Jordan S, Milde N, et al. Haemodynamic phenotypes and survival in patients with systemic sclerosis: the impact of the new definition of pulmonary arterial hypertension. Ann Rheum Dis 2020; 79: 370-378. 